\title{
A Multi-scalar Approach for the Modelling and Fabrication of Free-Form Glue-Laminated Timber Structures
}

\author{
Tom Svilans ${ }^{(\bowtie)}$, Paul Poinet, Martin Tamke, \\ and Mette Ramsgaard Thomsen \\ Centre for Information Technology and Architecture (CITA), KADK, \\ Copenhagen, Denmark \\ \{tsvi,paul.poinet\}@kadk.dk
}

\begin{abstract}
This research project presents both innovative multi-scalar modelling methods and production processes aimed at facilitating the design and fabrication of free-form glue-laminated timber structures. The paper reports on a research effort that aims to elucidate and formalize the connection between material performance, multi-scalar modelling (Weinan 2011), and early-stage architectural design, in the context of free-form glue-laminated timber structures. This paper will examine how the concept of multi-scalar modelling as found in other disciplines can also be used to embed low-level material performance of glue-laminated timber into early-stage architectural design processes, thus creating opportunities for feedback across the design chain and an increased flexibility in effecting changes. The research uses physical prototypes as a means to explore and evaluate the methods presented.
\end{abstract}

Keywords: Multiscale modelling • Glue-laminated timber · Material performance

\section{Introduction}

Timber as a building material is seeing a resurgence in research and architectural and construction industries. It is sustainable, renewable, and has many properties that make it a promising alternative to common building materials such as steel and concrete. New opportunities to expand and innovate with the use of engineered timber and free-form glulams in architecture emerge with recent advances in digital simulation tools and computational design workflows. Real progress in this area is, however, inhibited by issues concerning the integration of the multiple scales, material behaviours, and constraints from fabrication processes. We present innovative design, modelling, and fabrication workflows developed in an applied architectural and industrial context. We set a focus on approaches that benefit early-stage design in multi-disciplinary architectural practice, and on material performance as a key concern for industrial timber fabricators. These approaches must remain flexible and abstract enough to be used as early as possible within the design process, and to facilitate conversations and estimates before the scope of the design is even set. The following 
describes an effort towards this aim which integrates notions of multi-scalar modelling and material performance into the design and prototyping of an experimental free-form glue-laminated timber structure.

\section{Multi-scalar Modelling and Integrating Material Performance}

\section{New Design-to-Fabrication Frameworks}

One of the key aims of this research is to address the linearity in digital chain - from the design and development of an architectural proposal; its translation into material lengths, dimensions, and production data; and finally to its fabrication and assemblyby introducing opportunities for feedback, recursion, and bidirectionality into the process. This comes from two separate research projects, both part of the Innochain Training Network: one which looks at how the integration of material performance into early-stage architectural design can lead to new and better-informed methods of modelling and making free-form timber structures, and the other which investigates the notion of multi-scalar modelling as it applies to the design and management of complex architectural proposals. Both consider the separation and differences that exist between design and fabrication, and search for a way with which to close the gap and integrate the two ends of the architectural process. This rapprochement has been the subject of discussion in other areas, and theoretical frameworks and solutions have been proposed by other practitioners in the field. Indeed, the shift from linear and discrete modes of working between design and fabrication has also forced a reconsideration of the roles of architect and fabricator, enabled in large part by the accessibility and convergence of digital modelling tools with CAM software. The idea of the 'digital craftsman' (Scheurer 2013) has repurposed the contemporary architect/designer as a digitally-enabled designer/maker, made possible by the blurring between digital tools of design and digital tools of making. The familiarity with and integration of advanced modelling and simulation tools within contemporary design processes means that the production and manipulation of fabrication data is now more accessible than ever, and thus designers have the capacity to infuse their work with material and fabrication considerations from the very beginning. This feedback from production back to conception is also explored in the Fabrication Information Modelling (FIM) framework (Duro-Royo and Oxman 2015), which again ties the design of an architectural artefact to the processes and constraints of its making. Both frameworks seek to include fabrication parameters within the design process, though the risk is then that design decisions are driven and consumed by these low-level parameters and fabrication considerations.

\section{Multi-scalar Modelling}

Introduced within different fields - from mathematics to weather simulations - the concept of "Multi-Scalar Modelling" comes from the realization that the full behavior of a particular system cannot be represented within a single model, since important aspects of it transcend different scales and levels of resolution (Weinan 2011). 
Maintaining full detailed resolution through all levels within one single environment makes the system unwieldy, computationally expensive, and difficult to manage and change. Existing architectural design research projects have already introduced Multi-scalar Modelling as a design-to-fabrication paradigm where structures and data are linked across different scales through integrative pipelines and simulation frameworks that use specific design methods-from mesh-based bidirectional information flows (Nicholas et al. 2016), to graph-based modelling and relational networks (Poinet et al. 2016) — tackling the different issues raised above.

The multi-scalar approach in this research seeks also to mitigate those potential issues by introducing bidirectional feedback between the different scales of design. That is, design decisions made at a broad level affect the low-level parameters of individual components, but changes in low-level parameters can filter back up and affect higher-level aspects of the design. The goal is to not privilege either the top-down design-led path or the bottom-up fabrication-led path, but rather to achieve a mediated middle-ground where conflicts between scales can be resolved through changes at either level. The hope is that this design methodology allows more freedom in marrying fabrication realities to architectural design intentions, and a fluidity of change even later in the design-to-fabrication process.

The mechanism by which this multi-scalar method is explored in this research is an object-oriented data ecology and graph-based organizational models. This allows low-level timber-specific material feedback — such as bending limits, springback anticipation, and fabrication constraints - to be localized to individual elements or fabrication workpieces, while also being communicated to neighbouring elements and other components through relational graphs at higher scales. Similarly, optimizations which include many individual elements can be effected at higher scales, after which the specific changes can be communicated down to the individual element models, which in turn propagate those changes through their internal material model and verify it against constraints and material limits. This sort of back-and-forth communication between an element network and its constituent parts enables design decisions that can choose to either mitigate conflicts, or else override one scale in favour of the other in general or specific cases.

\section{Modelling and Data Management}

The design and modelling of a free-form glulam structure in such a feedback-laden context required a reconsideration of standard modelling tools. Moving from generic representational data types such as surfaces, curves, and meshes to more material-specific and constrained models meant that new corresponding data structures needed to be conceived and implemented, ones which would be able to describe and keep track of things such as material limits and fabrication parameters. This effort began as a set of simple convenience functions and data structures to ease the modelling process - analyses for enforcing bending limits, for example, or quick ways to generate oriented and dimensioned extrusions that would describe a free-form glulam blank. This cascaded into the implementation of a more generic and extensible class 
hierarchy which started to integrate more complex arrangements of glulams, machining features, and finer control over the geometric and material properties of the model. The main goal was to encapsulate as much information as possible into discrete objects and processes that had real counterparts - glulam blanks being the main object of interestand to separate the data from its representation - which means that geometry and visual feedback is generated on-demand, and most operations on or with these glulam data structures deal directly with the data, and not its geometrical output. These two simple but crucial considerations - encapsulation and the separation of data and representation - meant that the emerging library could remain lightweight-important, especially when handling a large number of free-form members - and modular. Variations or new forms of existing object types can be implemented through a simple class inheritance scheme, which opens up the use of the library for new and speculative glulam designs, as well as allowing it to be extended for specific project demands.

The data library mainly revolves around a generic Glulam data structure. From a multi-scalar point of view, this contains enough minimal information to keep track of individual lamellas within the Glulam, as well as lamella counts and dimensions that allow the total bounding volume and 3D representation to be generated. In such a way, the data type is kept compact and lightweight, while being capable of responding to analysis at different scales. This mentality extends to the generation and manipulation of Feature objects, which represent local relationships or properties, such as specific geometries on the glulam member or machining operations to be performed on it. The latter especially benefits from this minimalism, as toolpaths and cuts can be described more accurately and compactly by defining key workplanes and the parameters of a specific type of machining operation, for example. In this sense, it shares similarities with efforts such as the Building Transfer Language (BTL) (Stehling et al. 2014), which presents a way of describing timber machining operations in a compact, modular, and machine-agnostic way, without resorting to producing and interpreting geometrical models.

Therefore, by separating the properties of the model from its geometrical representation, it becomes trivial to link the model data to other models and inputs and thus dynamically drive the model from various scales and directions. Likewise, by encapsulating these properties within an object-oriented ecology of data types, objects retain these properties during transformations and changes to parts of the model. This is what allows the model to retain its relationships between individual members, material properties, and fabrication information during the design process. Keeping these types of operations and types discrete from each other also divides the complexity of the overall model into manageable and interchangeable chunks - an ecology of models that talk amongst themselves rather than a total whole.

\section{Prototyping and Fabrication}

\section{Demonstrator}

As a way to synthesize the multi-scalar concepts described earlier with the data management and software architecture described in the previous section, a free-form 
timber demonstrator was conceived and developed. This served as a case-study that allowed us to explore the proposed methods and evaluate their effectiveness as modelling and information-generating tools. The focus was on developing a modelling architecture that would be able to embody ideas of multi-scalar modelling down to the level of fabricating individual components. Improving the fabrication feasibility and precision is another on-going parallel effort. The demonstrator provided an opportunity to test the robustness of the software library and its flexibility once integrated into a multi-model and multi-resolution workflow. The criteria for success therefore focused more on the flexibility and communication between these models rather than a finished physical outcome.

As a design driver, a speculative branching glulam module was used to compose an enveloping structure (see Fig. 1). The branching module was the result of previous experiments with free-form glulams, taking advantage of timber bending limits as well as lamination techniques. It was devised as a way to create structural connections between more than two points into a single member through the process of lamination and cross-lamination, thereby creating a structural module which could be arranged into more complex and unique arrangements using simple end-to-end lap joints. With this modular branching in mind, a vaulted pavilion was conceived with a predefined branching pattern of structural members (see Fig. 2). The goal of the exercise was then to marry this predefined pattern to the material realities of the branching glulam member, while constraining it to our specific production capacities. This clash-between imposed pattern and material performance - was where the multi-scalar modelling framework was put to use. This feedback gave valuable direction for the final pavilion design: a vaulted structure between several foot conditions - a multi-legged catenary surface populated with the branching modules. At these foot conditions, where the weight of the whole pavilion was concentrated, structural members are thicker and fewer. As the structure expands from these foot conditions, the use of the branching module means that the structure divides and thins out, covering the vault surface in
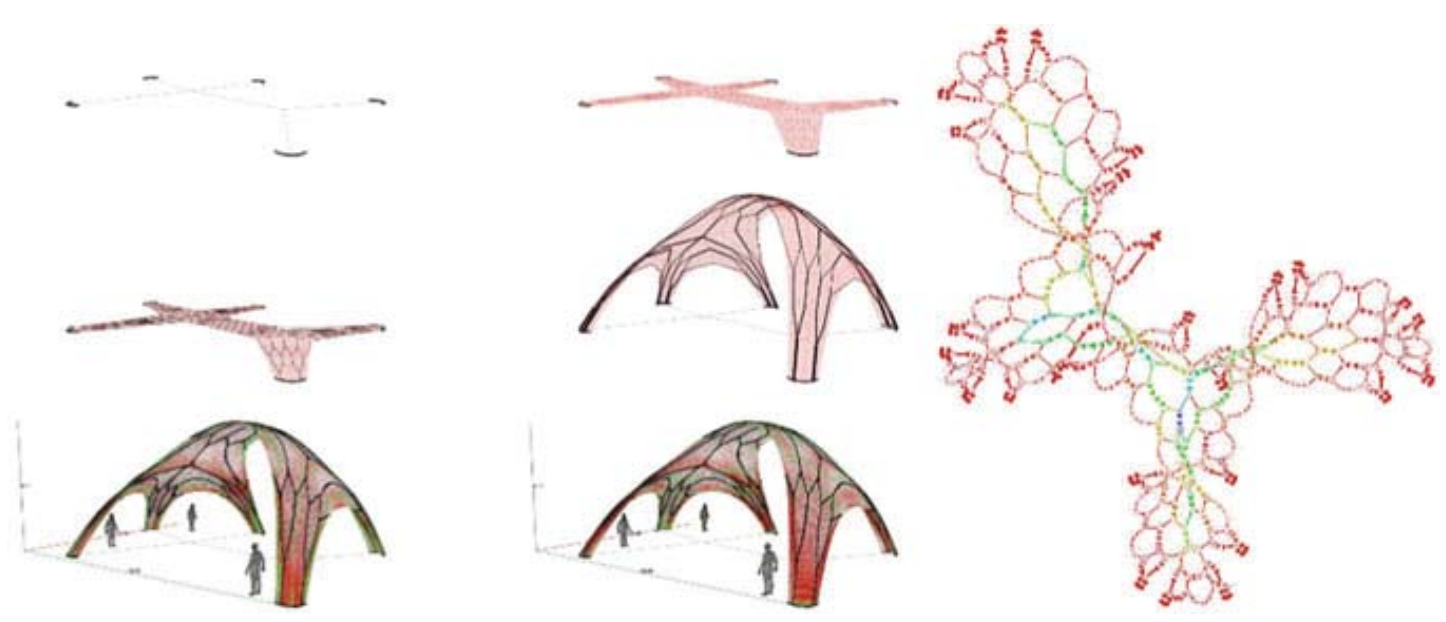

Fig. 1. Overview of the modelling process for a design iteration (left) and overall graph representation of the demonstrator (right). The color gradient corresponds to the degree of centrality of each component while the line thickness relates to the number of laminates contained within each member 

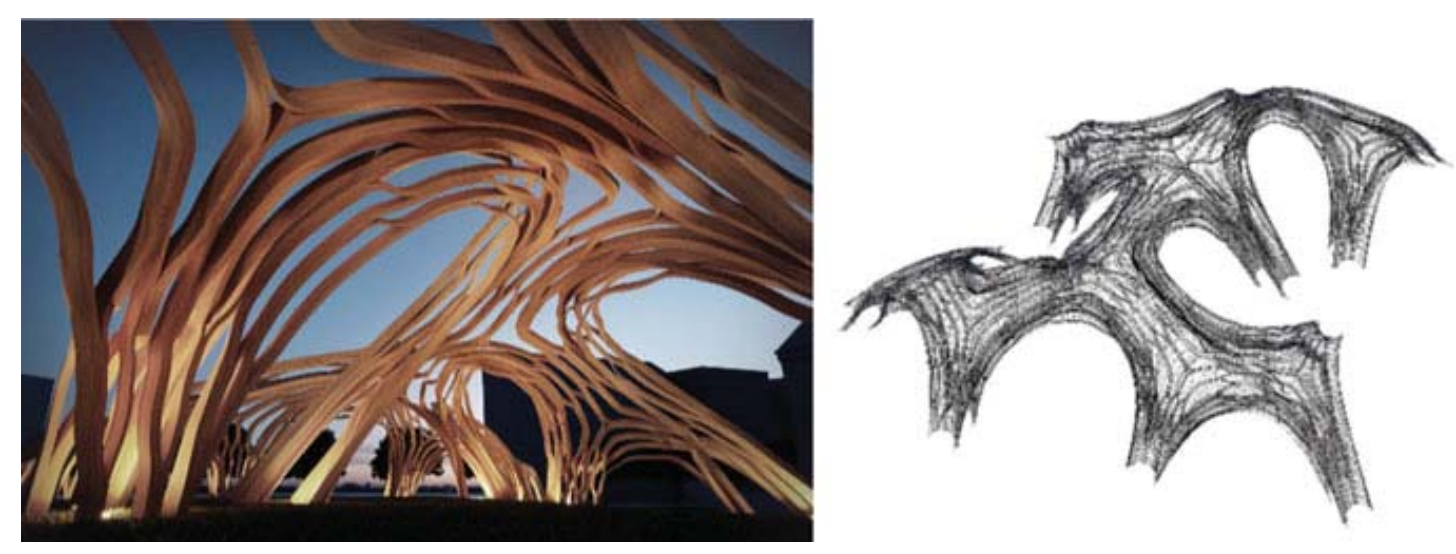

Fig. 2. Interior render of the demonstrator (left) and a graph representation of the whole design (right)

between with a network of thinner members. From this overall design, a number of individual components were brought through to physical prototyping as a way to explore the full cycle - from design to production - and explore the potential input of physical feedback into the multi-scalar model.

\section{Multi-scalar Modelling in Practice}

The architecture of the workflow was framed as a set of interconnected models operating at different scales (see Fig. 3). An undirected graph based on a mesh model at a broad level served to guide and organize the entire design-a schematic skeleton containing minimal but crucial information about the spatial location and orientation of each structural member, node, and the relationships between them. This allowed light-weight relaxation and optimization tools and structural analyses to be run over the whole network quite quickly, without delving into too much computational complexity or geometric detail. The constituent members of this network were then tied to individual beam models which began to describe each structural member in further geometric detail. Each beam model was linked to one or more material glulam models which contained information about the composition and type of glulam blank required to respond to the beam's structural and geometrical requirements. This dictated how the glulam blank would be formed: how tightly the wood fibres are aligned to the free-form beam axis influences the beams structural capacity, but has a consequent impact on the complexity of forming and constituent lamella sizes. This was then processed further into a fabrication model, which took into account fabrication limitations, specific fabrication processes and toolpath strategies, and so on. These processes were then looked at individually in terms of machine-specific workpiece fixation, accessibility of toolpaths, fabrication times, and other low-level fabrication parameters.

What this meant was that a design could be proposed and formulated into a graph, then tested through the lower-level models to yield the necessary types of glulam blanks, material dimensions, and fabrication data. More importantly, however, this allowed the aforementioned feedback between models. For example, a design iteration may cause a material model for a particular component to demand lamella sizes that are too small or impractical to use. Constraining these lamella dimensions to some other 


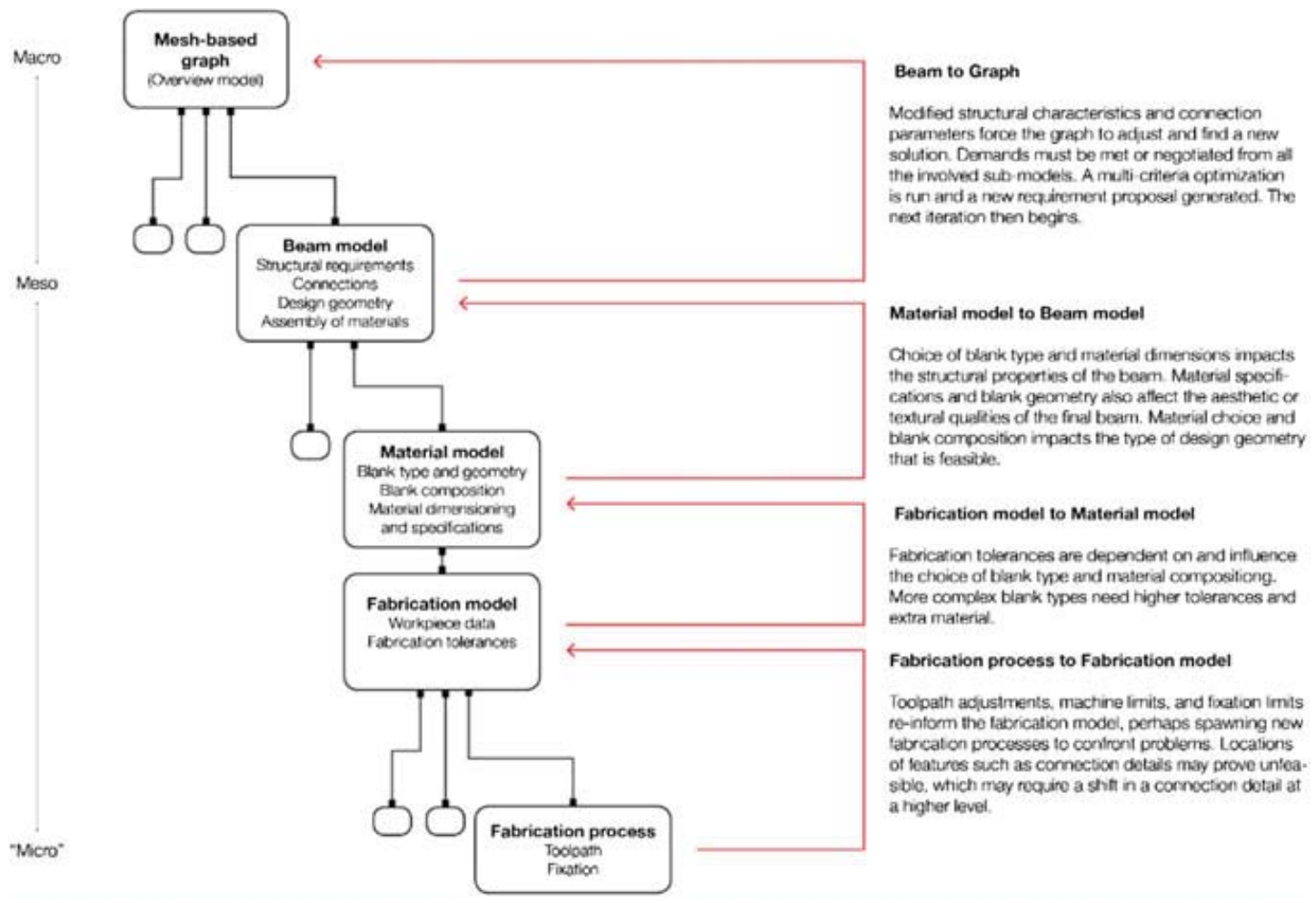

Fig. 3. Overview of the multi-scalar framework and the types of feedback between the different scales of models in this particular case

size could mean that a different type of glulam blank would have to be used for creating the module, which could have structural and geometric repercussions, and which further might force a re-evaluation of that module's role in the overall structural scheme and the roles of its neighbours (see Fig. 4). Estimation of timber quantities and dimensioning therefore became a quick byproduct of each design iteration, and the impact of design changes on fabrication time could be estimated at a glance. This also resulted in lower-level models also having an active voice in the mediation and development of the overall design scheme (Fig. 5).

\section{D Scanning}

As a way to bring physical feedback from the production process back into the model, each fabricated prototype was also 3D scanned. The role of scanning was on several levels: firstly and most obviously, it helped us compare the fabricated result with the production model, which reiterated the live and unpredictable behaviour of laminated wood (see Fig. 6). Secondly, it allowed us to locate the geometrically complex glulam blanks in front of the cutting spindle and adjust toolpaths and fixations as required. Thirdly - and possibly most interestingly for future work - as a documentation technique, the scanning allowed us to begin to record the deviations of the physical product from the fabrication model, and use this record to inform subsequent modelling decisions. In the most common case, seeing deviations caused by springback during the glulam blank forming process allowed us to adjust subsequent material models with greater bending to compensate for this springback. This raises questions about the 


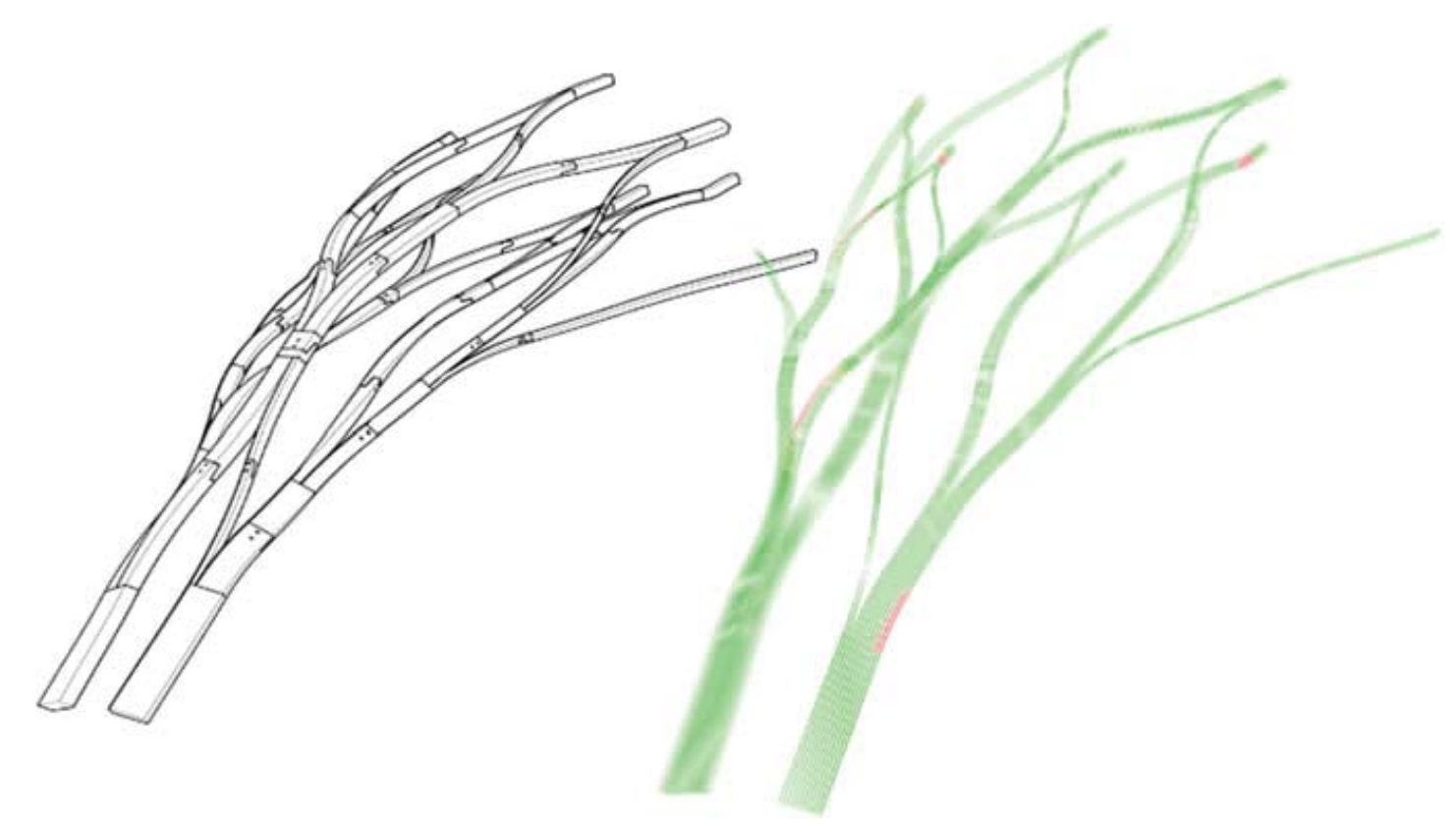

Fig. 4. The area around a foot condition extracted from the global design proposal, showing both geometric information from the beam model (left) and material information from the material model (right). In this case, the model is checked to see if the constituent lamellas of each module are within their bending limits

timeline of the design-to-production workflow and if it would be even possible to adjust larger design decisions when the first parts of the project have already been manufactured, and what the role of the accumulated scan feedback is once a particular project is over.

\section{Conclusion and Future Work}

In conclusion, as a design and information-generating framework, multi-scalar modelling is a promising alternative to other, more holistic or fragmented design methods, especially in the context of free-form timber. Designs that involve a complex and somewhat unpredictable material such as timber would greatly benefit from the feedback and low-level input that this method offers. Additionally, as a production and optimization tool, the presented workflow allowed a quick succession of design iterations, while avoiding a laborious translation from design information to fabrication information each time.

However, certain challenges remain, most notably of how this technique could better incorporate the glulam blank forming process and how it could help to tighten the tolerances of the finished products. This research is part of two larger research projects which look at both multi-scalar modelling and the role of material performance in glue-laminated timber construction. As such, future work will focus on addressing the integration of material performance in the forming and machining of glue-laminated components, and how feedback from this level could contribute and guide early-stage design decisions; as well as how physical feedback in a multi-scale design environment 

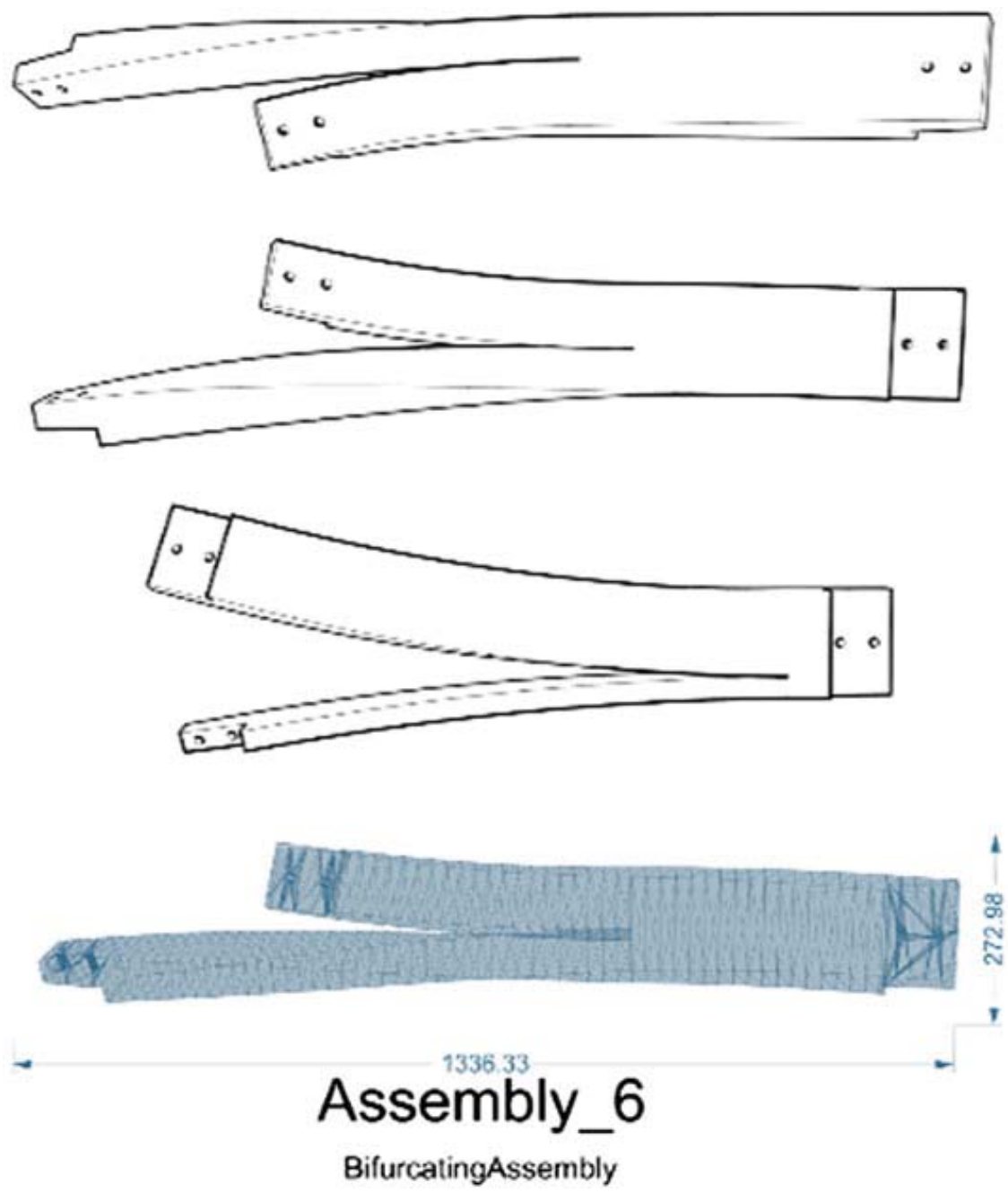

Fig. 5. The design broken down into individual component models

Fig. 6. Analysis of 3D scans of a bifurcating glulam blank (left) and its production geometry (right) 

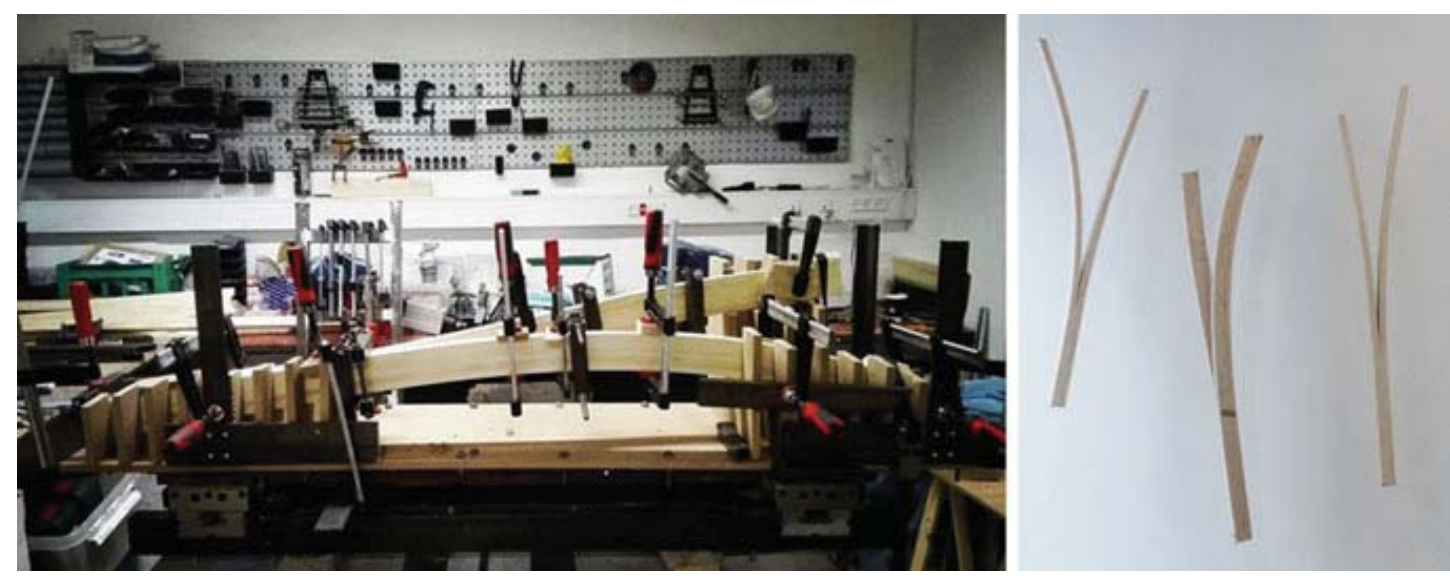

Fig. 7. Forming one of the bifurcating glulam components (left) and some finished examples (right)

could be recorded, accumulated, and leveraged for future use. Continued collaboration with the involved industry partners will allow this method to be tested against established workflows and methods of modelling, optimizing, and fabricating complex glue-laminated timber structures.

Most important, however, is the possibility of an informed mediation that this method enables: having multiple scales and resolutions available throughout a longer period during a project development phase allowed, in this case, a designer to make decisions faster and more frequently. By widening the scope of information available and modelling the interrelations between constraints at various scales, the method provides an increased measure of control over complexity in architectural design and points to ways in which free-form timber structures can become more feasible and accessible (Fig. 7).

Acknowledgements. This project was undertaken at the Centre for IT and Architecture, KADK, in Copenhagen, Denmark as part of the Innochain Early Training Network. This project has received funding from the European Union's Horizon 2020 research and innovation programme under the Marie Sklodowska-Curie Grant Agreement No. 642877. We would also like to express our sincere thanks to the CITAstudio Masters students that helped during the fabrication process.

\section{References}

Duro-Royo, J., Oxman, N.: Towards fabrication information modeling (FIM). In: MRS Proceedings, vol. 1800. Cambridge University Press (2015)

Nicholas, P., Zwierzycki, M., Stasiuk, D., Nørgaard, E., Thomsen, M.R.: Concepts and methodologies for multiscale modeling - a mesh-based approach for bi-directional information flows. In: ACADIA 2016-Posthuman Frontiers, pp. 110-129 (2016)

Poinet, P., Nicholas, P., Tamke, M., Thomsen, M.R.: Multi-scalar modelling for free-form timber structures. In: Proceedings of the IASS Annual Symposium, Spatial Structures in the 21st Century (2016)

Scheurer F (2013) Digital craftsmanship: from thinking to modeling to building. In: Marble S (ed) Digital Workflows in Architecture. Birkhäuser, Basel, pp. 110-129 
Stehling, H., Scheurer, F., Roulier, J.: Bridging the gap from CAD to CAM: concepts, caveats and a new Grasshopper plug-in. In: Gramazio, F., Kohler, M., Langenberg, S. (eds.) Fabricate: Negotiating Design \& Making, pp. 52-59 (2014)

Weinan E (2011) Principles of Multiscale Modeling. Princeton University, Princeton 